\section{Ist ein Diätversuch bei chronischer Urtikaria sinnvoll?}

Die chronische spontane Urtikaria ist sicherlich ein schwer zu behandelndes Krankheitsbild. Therapeutisch werden auch strenge Diäten empfohlen. Nach einer neueren Studie kann jedoch nur bei einem Drittel der betroffenen Patienten mit einer solchen Diät eine Besserung erreicht werden.

E ingeschlossen in die Studie wurden 140 Patienten mit chronischer spontaner Urtikaria, die mehr als sechs Wochen bestand. Die Patienten hatten auf die konventionelle Therapie mit Antihistaminika und Kortikoiden nicht ausreichend angesprochen. Sie erhielten eine strenge pseudoallergenfreie Diät über drei Wochen. Die Patienten ernährten sich vorzugsweise von frischen Lebensmitteln und vermieden konservierte Nahrungsmittel bzw. solche mit Zusatzstoffen.

Die Auswertung erfolgte entsprechend eines Scores, der die Urtikariaaktivität erfasste. 20 Patienten (14\%) zeigten ein deutliches Ansprechen, weitere 19 Patienten ein partielles Ansprechen auf die Diät. Darüber hinaus konnten neun $\mathrm{Pa}$ tienten ihre Medikation (Antihistaminika, Kortikoide) deutlich reduzieren, ohne dass es zu einer Verschlechterung der Symptomatik oder der Lebensqualität kam. Doch bei 22 Patienten (15\%) zeigte sich sogar eine Verschlimmerung des Krankheitsbildes.

Fazit: Bei einer Erkrankung mit individuell sehr unterschiedlichem Krankheitsverlauf ist eine Erfolgsrate von ca. 30\% nicht gerade sehr überzeugend. $\mathrm{Ob}$ man deshalb allerdings die pseudoallergenfreie Diät grundsätzlich als unwirksam abqualifizieren sollte, darüber lässt sich jedoch trefflich streiten. Auch darf man wohl annehmen, dass eine strenge pseudoallerdurchzuhalten und evtl. sogar ungesund ist, da bestimmte Früchte- und Gemüsesorten ebenfalls ausgeschlossen sind. Andererseits sollte man bei einem Krankheitsbild, das die Lebensqualität der Betroffenen stark beeinträchtigt, insbesondere dann, wenn keine überzeugende therapeutische Alternative zur Verfügung genfreie Ernährung langfristig kaum steht, alle therapeutischen Möglichkeiten so weit wie möglich ausschöpfen. Und die Patienten, die von einer solchen pseudoallergenreduzierten Diät profitieren, dürften Ihnen dankbar sein; denn wer heilt, hat immer Recht. P. Stiefelhagen

Magerl $M$ et al. Effects of pseudoallergenfree diet on chronic spontaneous urticaria: a prospective trial. Allergy 2010; 65:78-83

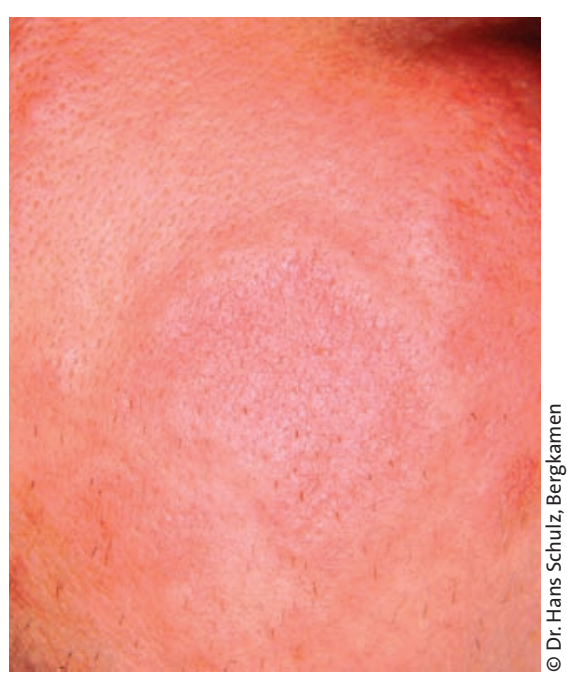

Urtikaria: Manchmal verschwindet sie unter einer Diät, sie kann aber auch schlimmer werden.

\title{
Psoriasis und schwanger - ein Problem?
}

Dass die Psoriasis mehr als ein Hautleiden ist, ist inzwischen gut bekannt. Was die Schuppenflechte für den Verlauf einer Schwangerschaft bedeutet, wurde bisher nicht systematisch untersucht. In dieser Studie prüfte man, ob Frauen mit Psoriasis mehr Risikofaktoren für ein negatives Schwangerschafts-Outcome haben als andere Schwangere.

D afür verwendete man Daten eines laufenden Projekts nordamerikanischer Teratologie-Spezialisten, das sich in einer prospektiven Kohortenstudie mit der Auswirkung von Autoimmunerkrankungen und deren Therapie auf die Schwangerschaft befasst. Für die vorliegende Analyse verglich man verschiedene Charakteristika schwangerer Teilnehmerinnen mit Psoriasis mit Schwangeren ohne Autoimmunleiden.

Erwartungsgemäß waren die Psoriatikerinnen vor der Schwangerschaft signifikant häufiger adipös $(\mathrm{p}<0,0001)$, sie rauchten häufiger $(\mathrm{p}<0,0001)$ und in ih- rer Gruppe gab es öfter die Diagnose Depression $(\mathrm{p}=0,03)$ als bei den anderen werdenden Müttern in der Studie. Außerdem nahmen sie präkonzeptionell seltener Vitamine ein $(p=0,004)$.

Mütterliche Adipositas ist nach Literaturdaten unter anderem mit einem erhöhten Risiko spezifischer kongenitaler Anomalien, mit Makrosomie, Hypoglykämie und niedrigen Apgar-Werten assoziiert. Eine Depression der Schwangeren geht mit einer höheren Wahrscheinlichkeit einer Frühgeburt, von niedrigem Geburtsgewicht und von Spontanaborten einher. Rauchen während der Schwangerschaft wurde ebenfalls mit einem reduzierten Geburtsgewicht sowie Fehlbildungen und Plazentakomplikationen in Zusammenhang gebracht. Alle genannten Risikofaktoren sind allerdings modifizierbar.

Fazit: Komorbiditäten sowie andere mit der Dermatose verbundene Faktoren könnten den Autoren zufolge ein Risiko für Schwangerschaftsverlauf und Kind bedeuten. - Ein weiteres Argument dafür, speziell Psoriasis-Patientinnen im gebärfähigen Alter umfassend auf Begleiterkrankungen und gesundheitlich relevante Gewohnheiten hin zu untersuchen und entsprechend zu betreuen.

wpa

Bandoli G et al. Potentially modifiable risk factors for adverse pregnancy outcomes in women with psoriasis. Br J Dermatol. 2010; 163: 334-9 